\title{
Exosomal miRNA423-5p mediated oncogene activity in papillary thyroid carcinoma: a potential diagnostic and biological target for cancer therapy
}

\author{
W. YE ${ }^{*}$ X. DENG ${ }^{*}$ Y. FAN ${ }^{*}$ \\ Department of General Surgery, Center of Thyroid and Parathyroid, Shanghai Jiao Tong University Affiliated Sixth People's Hospital, Shanghai \\ 200233, China \\ ${ }^{*}$ Correspondence: fanyouben2006@163.com \\ ${ }^{\#}$ Contributed equally to this work.
}

Received August 24, 2018 / Accepted December 19, 2018

\begin{abstract}
Exosomes are considered important "messengers" between cells and their cargo, and some of the miRNAs composing their cargo may be involved in oncogenic activity. Exosomal miRNA423-5p (Ex-miRNA423-5p) is upregulated in gastric cancer and associated with its poor prognosis. However, the role of exosome-derived miRNAs in papillary thyroid cancer (PTC) is still unclear and needs further study. In our work, we found that the level of Ex-miRNA423-5p was significantly increased in the serum of PTC patients compared with the serum of normal healthy people, and in vitro, we demonstrated that miRNA423-5p entered thyroid cancer cells through exosomes from thyroid cancer cell supernatant. Functional experiments demonstrated that overexpression of miRNA423-5p mimics in exosomes increased PTC cell migration and invasion, while the silencing of miRNA423-5p in TPC-1 cell exosomes inhibited PTC cell migration and invasion in vitro. These findings indicate that Ex-miRNA423-5p might serve as a potential tumor marker; thus, silencing miRNA423-5p in exosomes might represent a potential approach against PTC.
\end{abstract}

Key words: exosome, miRNA423-5p, papillary thyroid carcinoma, migration, invasion

Thyroid cancer is the most common endocrine tumor, accounting for approximately $3.8 \%$ of all newly diagnosed malignancies and approximately two thousand thyroid cancer patient deaths in the U.S. in 2016 [1-3]. Among the various types of thyroid cancer, papillary thyroid carcinoma (PTC) is the most common pathological type. The incidence of PTC is increasing year by year, accounting for more than $90 \%$ of all thyroid cancers [4]. PTC is well differentiated and belongs to relatively inert tumors. Most of the tumors are concealed and are characterized by slow progress and a good overall prognosis, but a small number of them show increased invasiveness. Even at the early stage, tumor invasion, lymph node metastasis, and lung, bone and other distant metastases can recur and metastasize after surgery [5-6]. Sometimes the metastases do not absorb iodine and are transformed into poorly differentiated or dedifferentiated tumors [7]. Therefore, the identification of benign and malignant nodules is particularly important. There is no specific clinical diagnosis or treatment for patients with unclear thyroid nodules. Therefore, the discovery of accurate and sensitive biomarkers that can reliably provide an early diagnosis of PTC has become important in oncology research in recent years.

Exosomes are involved in a type of intercellular communication that has been discovered in recent years [8-9]. They represent bilayer membrane vesicles with a diameter of 20-200 nm produced by the fusion of living cell multivesicular bodies with cell membranes [10]. MicroRNAs (miRNAs) carried by exosomes are advantageous in the diagnosis and tumor treatment because they contain only 21-25 base pairs of noncoding RNA and are protected by a bilayer membrane structure [10]. In recent years, it has been accepted that exosomes carrying miRNAs and secreted by tumor cells play an increasingly important biological role in the development of tumors [11]. In addition, unlike liposomes and other synthetic drug nanoparticle carriers, exosomes contain transmembrane and membrane-anchored proteins that may enhance endocytosis, thus promoting the delivery of their internal content [12].

However, the current research on exosomes in thyroid cancer is still in its infancy. Lee et al. demonstrated that exosomes carrying miRNA146 and miRNA222 are found in 
the culture supernatant of the thyroid cancer cell line TPC-1 [13]. Roman et al. found that miRNA21 and miRNA181a in plasma exosomes were significantly and differentially expressed in thyroid follicular tumors and PTC patients [14]. However, studies profiling the miRNA expression in PTC serum exosomes and the clinical diagnostic potential of specific miRNAs in PTC have not been reported. Some studies have indicated that miRNA423-5p targets the suppressor of fused protein to promote cancer growth and metastasis and serves as a novel biomarker for gastric cancer [15]. In addition, miRNA423-5p simultaneously contributes to a malignant phenotype and temozolomide chemoresistance in glioblastomas [16]. Quantitative real-time PCR (qRT-PCR) of serum exosomes from PTC patients showed that Ex-miRNA423-5p was significantly elevated and that it is closely related to lymph node metastasis. Furthermore, Ex-miRNA423-5p significantly promoted PTC cell migration and invasion. Therefore, our work provides a new basis for PTC diagnosis and treatment.

\section{Materials and methods}

Serum collection. Serum from paired PTC patients (with papillary thyroid carcinoma but without other tumor types and other types of diseases, including cardiovascular disease, high blood pressure and diabetes) and healthy donors (without papillary thyroid carcinoma, other tumor types and other types of diseases, including cardiovascular disease, high blood pressure and diabetes) was obtained from 60 patients who underwent surgical resection at the Department of Thyroid Surgery, Sixth People's Hospital, School of Medicine, Shanghai Jiao Tong University (Shanghai, China). Each patient signed a written informed consent form, and the study was approved by the Ethics Committee of Shanghai Jiao Tong University Affiliated Sixth People's Hospital. Patients enrolled in this study did not receive any treatment before surgery. Blood samples were collected and centrifuged at $3000 \mathrm{rpm}$ for $15 \mathrm{~min}$ at $4^{\circ} \mathrm{C}$ to obtain serum, which was removed, placed into a new tube and stored at $-80^{\circ} \mathrm{C}$ until use.

Cell culture and supernatant collection. The PTC cell lines KTC- 1 and TPC- 1 were cultured in RAPI-1640 supplemented with $10 \%$ fetal bovine serum (FBS), $100 \mathrm{U} / \mathrm{ml}$ penicillin, and $100 \mu \mathrm{g} / \mathrm{ml}$ streptomycin. Cells were incubated in a humidified chamber with $5 \% \mathrm{CO}_{2}$ at $37^{\circ} \mathrm{C}$ and regularly tested for mycoplasma (MycoProbe Mycoplasma Detection Kit, R\&D Systems). When cells reached a density of $60-70 \%$, the medium was replaced with FBS-free 1640 medium. After starvation for $48 \mathrm{~h}$, the cell supernatant was collected and stored at $-80^{\circ} \mathrm{C}$ until use.

Exosome isolation. Exosomes were isolated from the serum and supernatant of PTC cells according to the experimental method described in previously published studies [17-18]. In brief, cells were centrifuged for $30 \mathrm{~min}$ at 2000 $\mathrm{g}$ and $4{ }^{\circ} \mathrm{C}$, the supernatant was collected and centrifuged for $30 \mathrm{~min}$ at $10000 \mathrm{~g}$ and $4^{\circ} \mathrm{C}$, and finally, the obtained super- natant was centrifuged for $70 \mathrm{~min}$ at $100000 \mathrm{~g}$ and $4^{\circ} \mathrm{C}$. The pellet was resuspended in $2-3 \mathrm{ml}$ of $1 \times \mathrm{PBS}$, and the sample was filtered using $0.22 \mu \mathrm{m}$ pore filters. Next, the sample was centrifuged for $70 \mathrm{~min}$ at $100000 \mathrm{~g}$ and $4{ }^{\circ} \mathrm{C}$. The resulting pellet was resuspended in $50-100 \mathrm{ml}$ of $1 \times$ PBS.

Exosome identification by transmission electron microscopy and nanoparticle tracking analysis. Twenty $\mathrm{ml}$ of exosomes were added onto Formvar carbon-coated copper grids and allowed to adsorb for $10 \mathrm{~min}$ before the excess fluid was drained. Then, the adsorbed exosomes were negatively stained with $2 \%(\mathrm{w} / \mathrm{v})$ phosphotungstic acid $(\mathrm{pH}$ 6.8) for $5 \mathrm{~min}$, air-dried under an electric incandescent lamp, and analyzed with a transmission electron microscope (FEI Tecnai 12, Philips). Exosomes were diluted with $700 \mathrm{ml}$ of PBS and thoroughly mixed. The size and concentration of exosomes were measured using Nanoparticle Tracking Analysis. A NanoSight LM10 instrument (Malvern Instruments) with a $405 \mathrm{~nm}$ laser-equipped sample chamber was used according to the manufacturer's instructions.

RNA extraction, qRT-PCR and miRNA sequencing library. Ex-miRNAs in the culture supernatants and serum were extracted using the miRNeasy Serum/Plasma Kit (Qiagen, Germany) according to the manufacturer's instructions. The extracted miRNAs were reverse transcribed using the miScript II RT Kit (Qiagen, Germany) and detected using the miScript SYBR Green PCR Kit (Qiagen, Germany). The relative Ex-miRNA423-5p expression was normalized to miR-39 expression (Qiagen, Germany). To calculate the relative expression of serum Ex-miR-423-5p in PTC patients, the $2^{-\Delta \mathrm{Ct}}$ method was used $(\Delta \mathrm{Ct}=\mathrm{CtmiR}-423-5 \mathrm{p}$ CtmiR-39), and the relationship between healthy donors and PTC patients was evaluated. The miRNA423-5p primer (5'-TGAGGGGCAGAGAGCGAGACTTT-3') was supplied by Shenggong (Shanghai, China). The total RNA from each sample was used to prepare the miRNA sequencing library, which included the following steps: 1) 3'-adaptor ligation; 2) 5'-adaptor ligation; 3) cDNA synthesis; 4) PCR amplification; 5) size selection of $\sim 135-155$ bp PCR-amplified fragments (corresponding to $\sim 15-35$ nt small RNAs). The samples in the library were denatured as single-stranded DNA molecules, captured on Illumina flow cells, amplified in situ as clusters and finally sequenced for 50 cycles on Illumina NextSeq according to the manufacturer's instructions.

Western blotting. Exosomes were harvested, cells were lysed in RIPA lysis buffer (Beyotime, Shanghai, China), and protein concentration was measured by a BCA kit (Beyotime). Twenty micrograms of protein were loaded on $12 \%$ sodium dodecyl sulfate-polyacrylamide gel electrophoresis then transferred to a polyvinylidene difluoride membrane (PVDF; Millipore, Boston, MA, USA). After blocking with $5 \%$ nonfat milk for $1 \mathrm{~h}$, the PVDF membrane was incubated with monoclonal primary antibody (TSG-101, Abcam, Cambridge, UK) at $4{ }^{\circ} \mathrm{C}$ overnight and then secondary antibody (Cell Signaling Technology, Shanghai, 
China) for another $1 \mathrm{~h}$ at room temperature. Target protein bands were visualized using enhanced chemiluminescence reagents (Pierce, Rockford, IL).

MiRNA electroporation into exosomes. Exosomes were ultracentrifuged according to the protocol described above. The pellet was suspended in $100 \mathrm{ml}$ of LONZA solution V (LONZA, Germany) then 20 of ml Cy3-miRNA 423-5p was added and mixed. After the LONZA X-001 electroporation procedure, the solution was transferred into a new centrifuge tube and immediately placed on ice. Four $\mathrm{ml}$ of PBS and $3 \mathrm{ml}$ of DiO (BIYUNTIAN, Nanjing, China) were added to the solution, which was then incubated for $30 \mathrm{~min}$ at room temperature. Next, ultracentrifugation was performed for $70 \mathrm{~min}$ at $110000 \mathrm{~g}$ and $4^{\circ} \mathrm{C}$. After the supernatant was removed, the pellet was suspended in $100 \mathrm{ml}$ of 1640 medium and co-cultured with 15000 cells on 24-well plate slides. After $4 \mathrm{~h}$ of incubation, cells were fixed and stained with DAPI (Sigma, USA). Cell morphology was observed under a confocal microscope (Leica, Germany).

Migration and invasion assays. After the electroporation of the miRNA423-5p mimics and inhibitor (RUIBO, Guangzhou, China) into exosomes, the exosomes were then co-cultured with PTC cells as described above for $48 \mathrm{~h}$. In the migration assay, $1.5 \times 10^{4}$ cells were placed on the top chamber of each insert (BD Biosciences, NJ, USA) with a noncoated membrane. In the invasion assay, $2 \times 10^{4}$ cells were placed on the upper chamber of each Matrigel-coated insert (BD Biosciences, Billerica, MA, USA). After $18 \mathrm{~h}$ incubation at $37^{\circ} \mathrm{C}$, the upper chamber cells that either migrated or invaded were fixed and stained in a dye solution containing $0.1 \%$ crystal violet and $20 \%$ methanol. The number of cells that either migrated or invaded was counted and images were taken using an IX71 inverted microscope (Olympus Corp, Tokyo, Japan).

Statistical analysis. Statistical analysis was performed using SPSS 16.0 software (SPSS, Inc., Chicago, IL). The results are presented as the mean \pm standard deviation (SD). Data were analyzed using the Student's t-test (two-tailed) unless otherwise specified (paired t-test, $\chi^{2}$ test or Spearman correlation). A p-value of $<0.05$ was considered statistically significant.

\section{Results}

Separation and identification of exosomes. Exosomes isolated from the serum of PTC patients and healthy controls and from the supernatant of PTC cells exhibited a spherical or elliptical vesicle shape, as shown by transmission electron microscopy (Figures 1A, 1D). Nanoparticle tracking analysis was used with an exosome diameter distribution ranging from $20-200 \mathrm{~nm}$ (Figures 1B, 1E). Next, the proteins extracted from the exosomes were collected, and western blotting showed that a specific protein marker, TSG101, was expressed in the exosomes (Figures 1C, 1F). These results indicated the successful separation and identification of the exosomes.

MiRNA423-5p is upregulated in PTC serum exosomes. Noncoding RNAs play an important role in tumor invasion
A

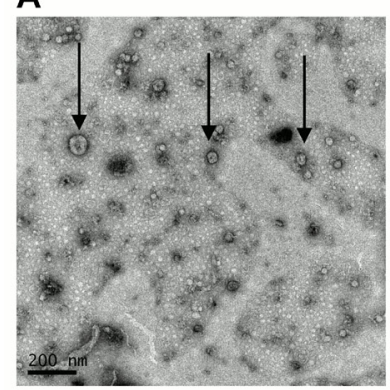

D

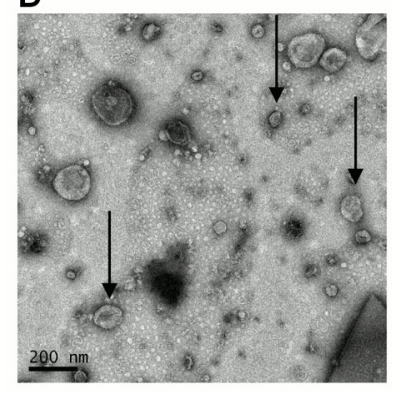

B

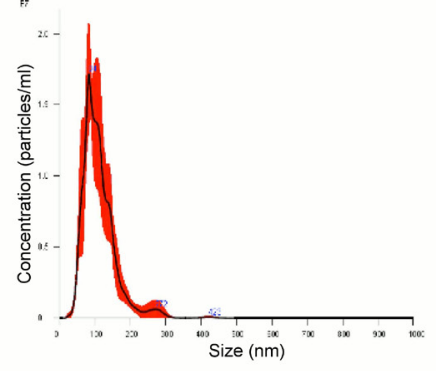

E

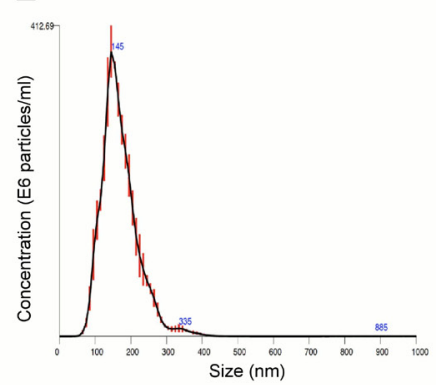

C

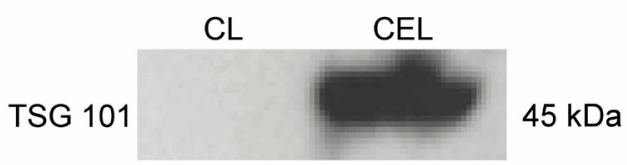

$\mathbf{F}$

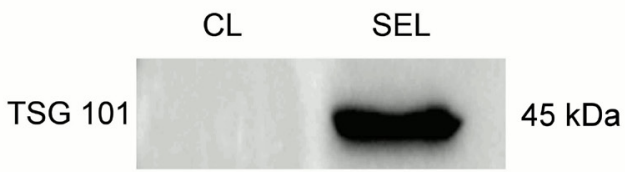

Figure 1. Separation and identification of exosomes. Exosomes derived from TPC-1 cell supernatant (A) and PTC serum (D) were characterized by transmission electron microscopy (TEM). Scale bar, $200 \mathrm{~nm}$. TPC-1 cell supernatant (B) and PTC serum (E) by nanoparticle tracing analysis (NTA). Scale bar, $100 \mathrm{~nm}$. Characterization of TPC-1 cell-derived exosomes $(\mathrm{C})$ and PTC serum exosomes (F) by detecting the exosomal membrane marker TSG101 (CL: cell lysate, CEL: cell exosome lysate, SEL: serum exosome lysate). 
and metastasis, and according to the literature, the most abundant content in exosomes is miRNA. Therefore, to explore the expression of miRNAs in PTC serum exosomes, we collected the serum from 3 healthy donors (control) and 3 PTC patients (PTC). Exosomes were isolated and identified as described above, and RNAseq analysis was performed. We focused on miRNAs that had a $>2^{2}$ - or $<2^{-2}$-fold change in both groups. For specific expression of differential miRNA expression, please refer to Table S1. Eleven miRNAs that exhibited a significantly different expression between the two groups were identified. Among them, hsa-miRNA23-5p (miRNA423-5p) was the most abundant miRNA in the serum of the PTC group (Figure 2A). Next, we enhanced our investigation by extending the analysis to the serum from 30 healthy donors and 60 PTC patients. The total RNA from the exosomes was extracted, qRT-PCR was performed, and the results showed that Ex-miRNA423-5p was significantly increased in PTC patients (Figure 2B) and closely associated with lymph node metastasis (Figure 2C). These results showed that miRNA423-5p was upregulated in PTC serum exosomes, suggesting that Ex-miRNA423-5p might serve as a potential tumor biomarker in PTC patients.

MiRNA423-5p is delivered into PTC cell lines via PTC cell-secreted exosomes. To further investigate whether miRNA423-5p enters PTC cell lines through exosomes secreted by PTC cell lines, PTC cell supernatant was collected, and exosomes were extracted. The cy3-labeled miRNA423-5p entered DiO-labeled exosomes by electroporation. After ultracentrifugation, the exosomes containing the cy3-labeled miRNA423-5p were co-cultured with TPC-1 cells for $4 \mathrm{~h}$. The results showed red and green staining inside TPC-1 cells due to cy3-miRNA423-5p and DiO-labeling, respectively (Figure $3 \mathrm{~A}$, left panel). Without electroporation, TPC-1 cells co-cultured with DiO-labeled exosomes containing cy3-miRNA423-5p did not show any red staining (Figure 3A, middle panel). TPC-1 cells that were neither co-cultured with DiO-labeled exosomes nor electrophoresed did not show any red or green color (Figure 3A, right panel). After $48 \mathrm{~h}$ of co-culture incubation, the RNA was extracted from the co-cultured cells, and reverse transcription and qRT-PCR were performed. The results showed that the level of miRNA423-5p was significantly increased in PTC cells (Figure 3B, left panel), but no difference was observed in the other two treatments, shown in the other two panels (Figure 3B, middle panel; Figure 3B, right panel). Based on these experimental results, it could be concluded that miRNA423-5p entered the PTC cells through exosomes secreted by the PTC cells.

Overexpression of miRNA423-5p into exosomes promotes the migration and invasion of PTC cells in vitro. Invasion and migration experiments in vitro showed that the overexpression of Ex-miRNA423-5p mimics in exosomes significantly promoted the migration and invasion of KTC-1 and TPC-1 (Figures 4A, 4B), while Ex-miRNA423-5p inhibitors in exosomes inhibited the migration and invasion of KTC- 1 and TPC-1 (Figures 4C, 4D). These results suggested that PTC exosomes could deliver miRNA 423-5p into PTC cell lines and promote their migration and invasion in vitro.
A

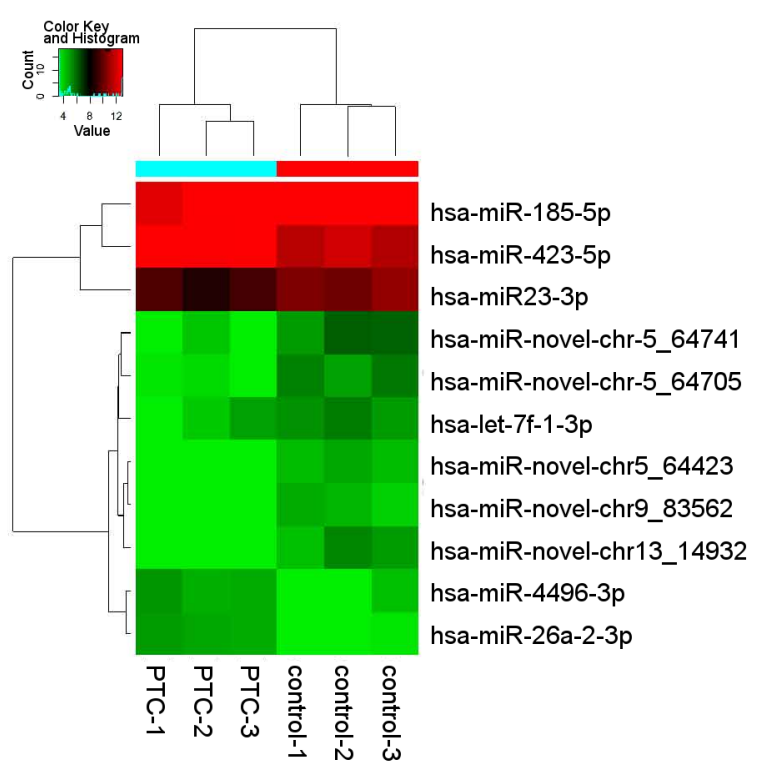

B

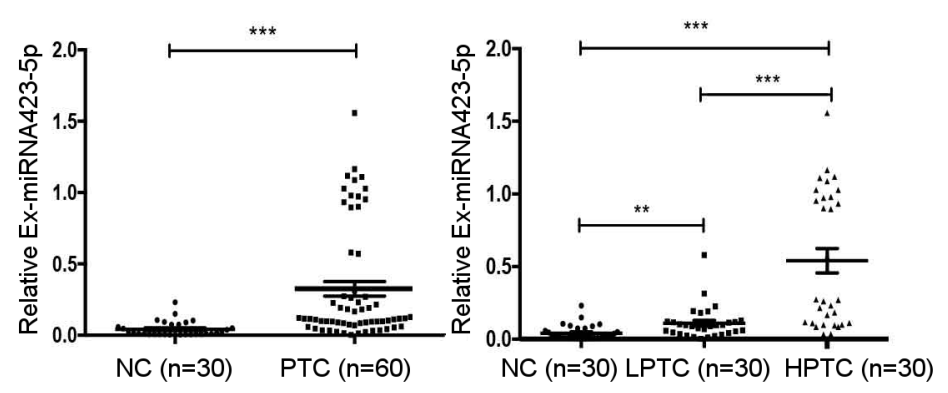

Figure 2. MiRNA423-5p is upregulated in PTC serum exosomes. A) A hierarchical clustering analysis of 11 miRNAs that were differentially expressed in 3 paired samples of PTC patients and corresponding healthy donors (fold change $>2^{2}$ or $<2^{-2}, p<0.05$ ). Expression values are represented in shades of red and green. B) The Ex-miRNA423-5p expression in healthy donors (Control) and PTC patients (PTC). C) The Ex-miRNA423-5p expression in PTC patients was closely associated with lymph node metastasis. The results are presented as the mean $\pm \mathrm{SD}$. ${ }^{\star *} \mathrm{p}<0.01,{ }^{* * *} \mathrm{p}<0.001$. (NC: healthy donors, LPTC: PTC without lymph node metastasis, HPTC: PTC with lymph node metastasis). 


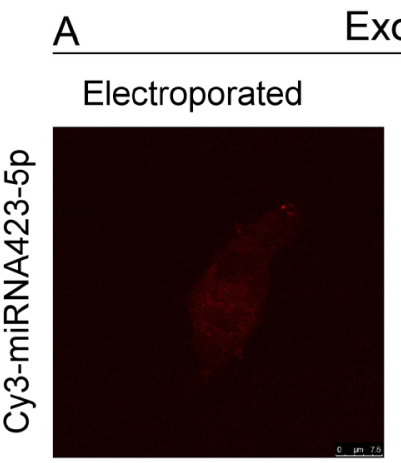

\section{Exosome}
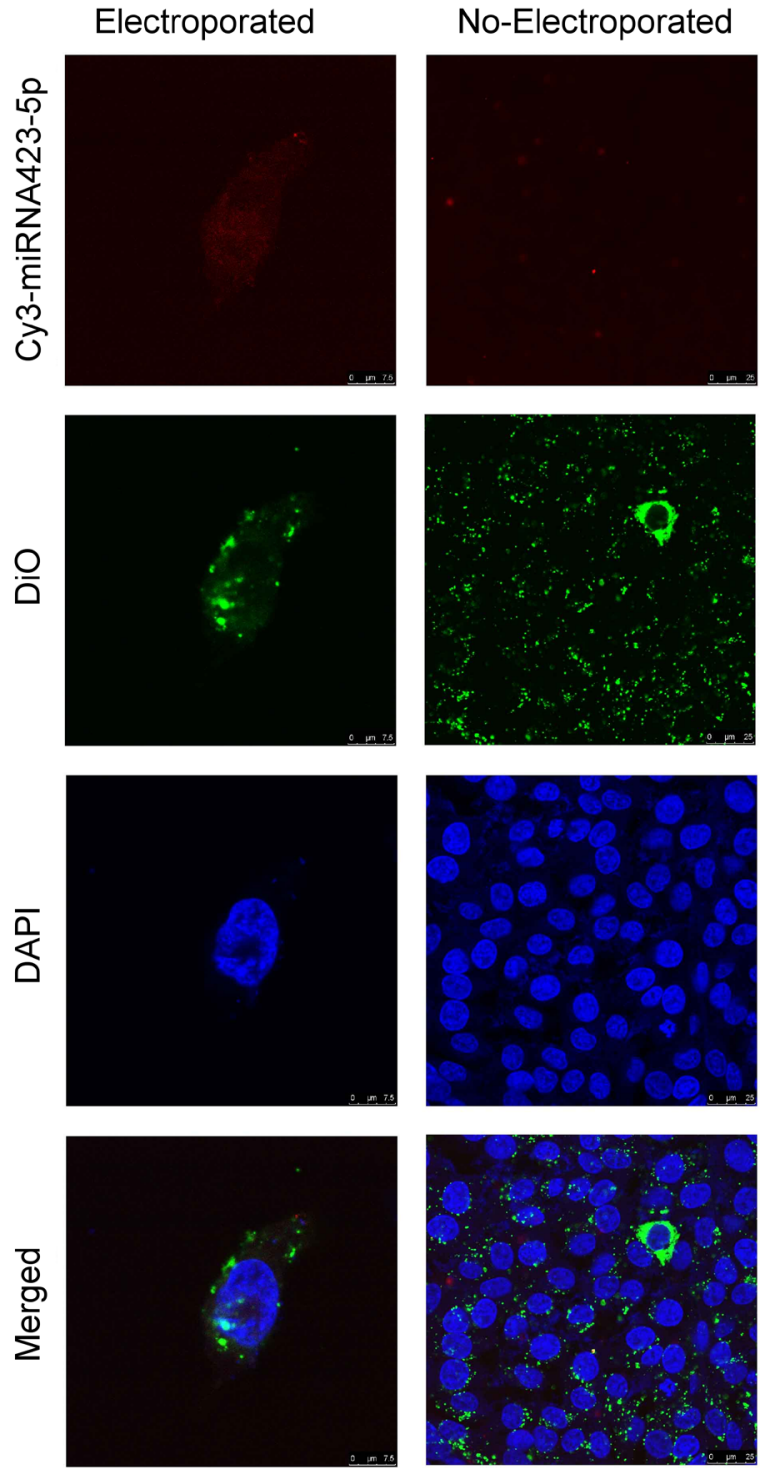

left panel
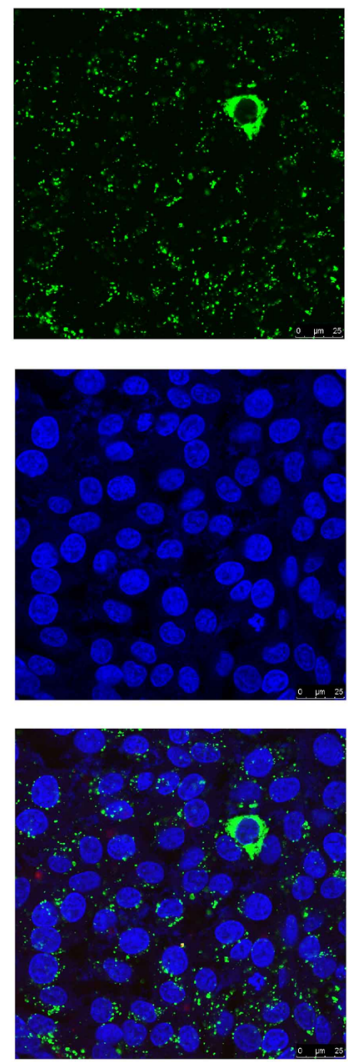

middle panel

\section{No-Electroporated}
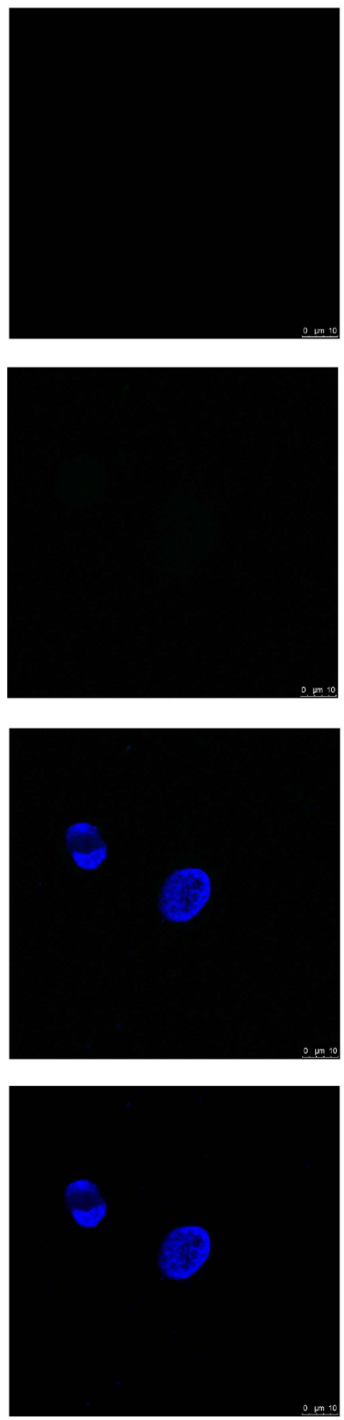

right panel
B

left panel

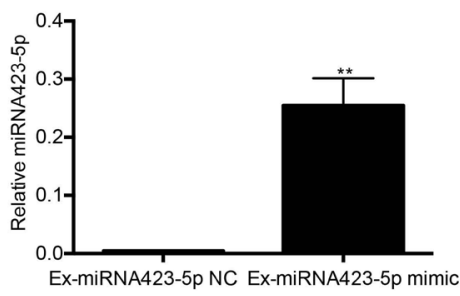

middle panel

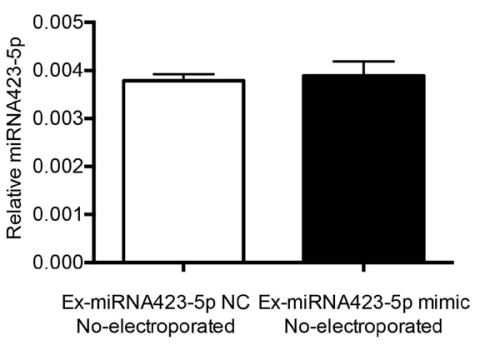

right pane

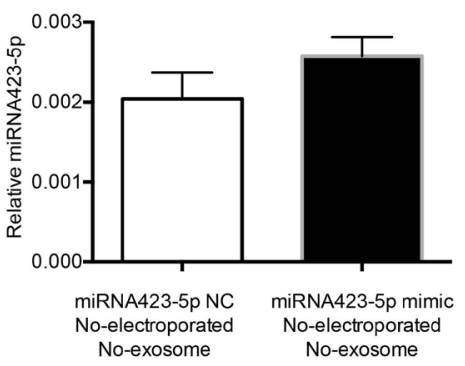

Figure 3. MiRNA423-5p is delivered into PTC cell lines via PTC cell-secreted exosomes. A) Left panel: Cy3-labeled miRNA423-5p (red) into DiOstained TPC-1 cell exosomes by electroporation (green) and co-cultured with TPC-1 cells; middle panel: Cy3-labeled miRNA423-5p and DiO-stained TPC-1 cell exosomes (green) without electroporation, co-cultured with TPC-1 cells; right panel: Cy3-labeled miRNA423-5p co-cultured with TPC-1 cells. B) Left panel: miRNA423-5p was significantly increased in TPC-1 cells, but no difference was observed in the other two panels (middle panel, right panel).

\section{Discussion}

An accurate understanding of the key molecules involved in the development of PTC is essential for the effective control of the disease. Although the diagnostic accuracy of PTC has improved due to the popularity of ultrasound technology, no effective molecular diagnostic biomarkers are currently available [4]. Previous reports indicate that
miRNAs carried by exosomes can be used not only as molecular markers for tumor diagnosis and prognosis, but exosomes can also be used as genetic engineering vectors to carry siRNA or shRNA targeting specific oncogenes [19-20]. In this study, we demonstrated that Ex-miRNA23-5p significantly promoted the migration and invasion of PTC cells in vitro, providing a new approach for the diagnosis and treatment of PTC. 

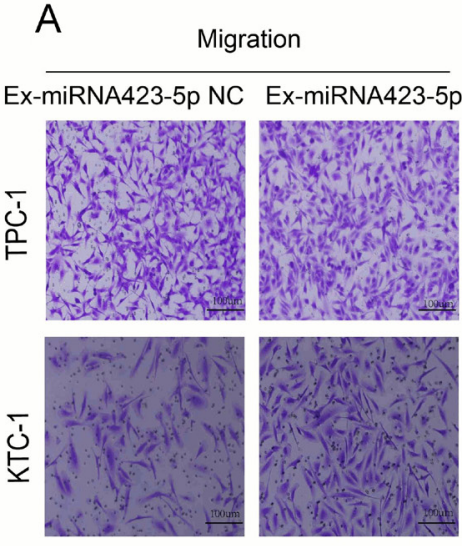

C

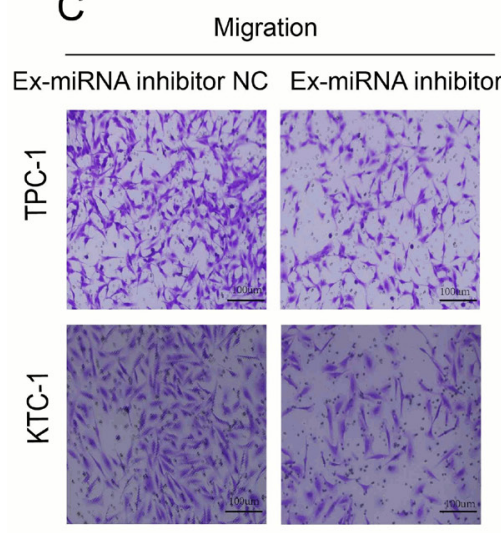

Ex-miRNA423-5p
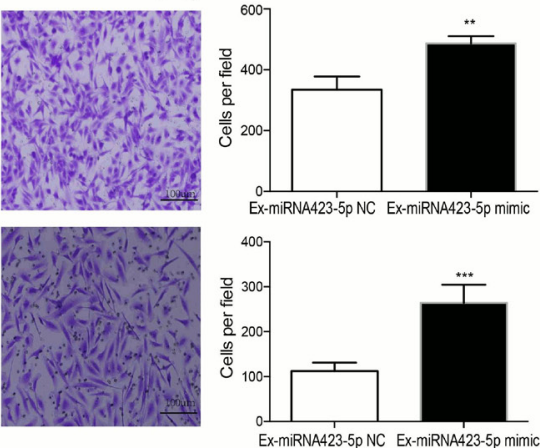

B

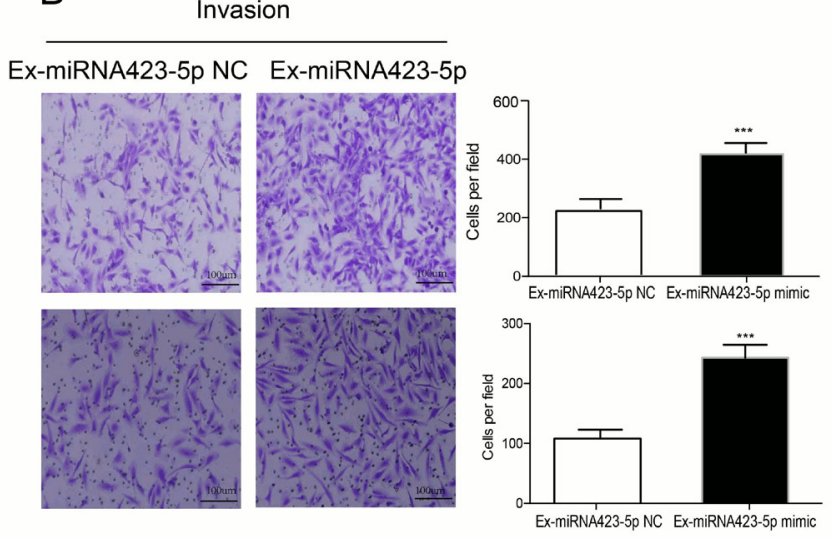

D

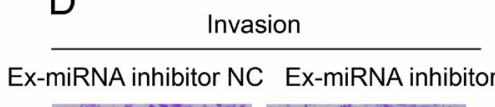

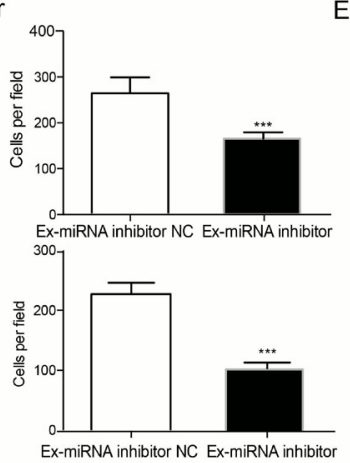
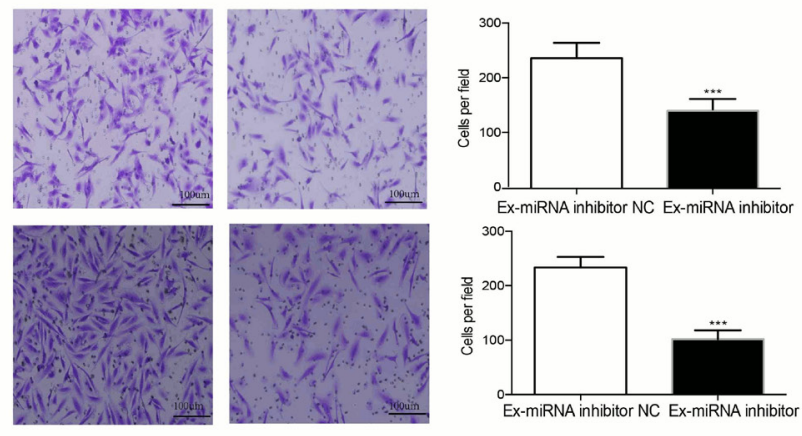

Figure 4. Overexpression of miRNA423-5p into exosomes promotes the migration and invasion of PTC cells in vitro. Migration (A) and invasion (B) of TPC-1 and KTC-1 cells with TPC-1 cell Ex-miRNA423-5p mimics. Target silencing of miRNA423-5p in TPC-1 cell exosomes inhibited the migration (C) and invasion (D) of TPC-1 and KTC-1 cells in vitro. Representative images are shown on the left, and the average number of cells per field at the indicated time points is shown on the right. The results are presented as the means $\pm \operatorname{SD} .{ }^{*} \mathrm{p}<0.05,{ }^{\star *} \mathrm{p}<0.01$, and ${ }^{\star * *} \mathrm{p}<0.001$.

In the current study, we first discovered the upregulation of Ex-miRNA423-5p in PTC patients, and then we further demonstrated that this upregulation was closely associated with lymph node metastasis. Therefore, we speculated that Ex-miRNA423-5p might be a molecular biomarker of PTC. These results are consistent with a previous report showing that miRNA423-5p targets the suppressor of fused protein to promote cancer growth and metastasis and serves as a novel marker for gastric cancer [15]. Our data also showed that PTC prognosis through Ex-miRNA423-5p evaluation is reliable, although further studies would be required to provide more supporting data.

Another aim of this study was to confirm our hypothesis that the exosomes carry the miRNA as a messenger of cell communication and that they can transfer this information between the cells. Our experiments demonstrated that Ex-miRNA423-5p could translocate into cells when co-cultured with exosomes carrying Ex-miRNA423-5p inserted by electroporation. This finding is consistent with a previous report showing exosome-mediated delivery of siRNA in vitro and in vivo [19]. Previous studies on gastric cancer cells and glioblastoma showed that miRNA23-5p regulates cell proliferation and invasion by targeting trefoil factor 1, contributing to a malignant phenotype and temozolomide chemoresistance [16]. In this study, we demonstrated that the overexpression of Ex-miRNA423-5p promoted the migration and invasion abilities of PTC cells [20].

In addition, an increasing number of studies have shown that exosomes containing miRNA can be used as carriers for nanomaterials, which have the function of transporting drugs or transmitting cell signals [21-23]. We also showed that exosomes could be used as siRNA drug carriers to silence miRNA423-5p in the treatment of PTC. Indeed, our results demonstrated that exosomes carrying the silenced miRNA423-5p inhibited the migration and invasion of PTC cells. These data indicated that Ex-miR-423-5p might be a potential biological target for PTC, although in vivo evidence is necessary to confirm our results and to confirm the soluble specificity of exosomes to recognize PTC cells. 
Overall, our research demonstrated that Ex-miRNA423-5p was upregulated in PTC patients and was closely associated with lymph node metastasis. More significantly, we offered promising evidence that exosomes secreted by the PTC cell line could deliver miRNA423-5p into PTC cells and promote PTC cell migration and invasion in vitro. The silencing of miRNA423-5p in exosomes inhibited this function of PTC cells in vitro. Therefore, our study revealed that Ex-miRNA23-5p might be considered a potential biomarker and therapeutic target in PTC. Future challenges include identifying additional Ex-miRNA423-5p targets, which will further elucidate its function in PTC.

Supplementary information is available in the online version of the paper.

Acknowledgements: This research was supported by the preresearch fund of Shanghai Jiao Tong University Affiliated Sixth People's Hospital (LYNYLC-0049) and National Natural Science Foundation of China (Grant No. 81670718).

\section{References}

[1] KITAHARA CM, SOSA JA. The changing incidence of thyroid cancer. Nat Rev Endocrinol 2016; 12: 646-653. https:// doi.org/10.1038/nrendo.2016.110

[2] SIEGEL RL, MILLER KD, JEMAL A. Cancer Statistics, 2017. CA Cancer J Clin 2017; 67: 7-30. https://doi.org/10.3322/ caac. 21387

[3] PELLEGRITI G, FRASCA F, REGALBUTO C, SQUATRITO S, VIGNERI R. Worldwide increasing incidence of thyroid cancer: update on epidemiology and risk factors. J Cancer Epidemiol 2013; 2013: 965212. https://doi. org/10.1155/2013/965212

[4] HAUGEN BR, ALEXANDER EK, BIBLE KC, DOHERTY GM, MANDEL SJ et al. 2015 American Thyroid Association Management Guidelines for Adult Patients with Thyroid Nodules and Differentiated Thyroid Cancer: The American Thyroid Association Guidelines Task Force on Thyroid Nodules and Differentiated Thyroid Cancer. Thyroid 2016; 26: 1-133. https://doi.org/10.1089/thy.2015.0020

[5] GUILMETTE J, NOSE V. Hereditary and familial thyroid tumours. Histopathology 2018; 72: 70-81. https://doi. org/10.1111/his.13373

[6] XING M. Molecular pathogenesis and mechanisms of thyroid cancer. Nat Rev Cancer 2013; 13: 184-199. https://doi. org/10.1038/nrc3431

[7] RIESCO-EIZAGUIRRE G, SANTISTEBAN P. ENDOCRINE TUMOURS: Advances in the molecular pathogenesis of thyroid cancer: lessons from the cancer genome. Eur J Endocrinol 2016; 175: R203-217. https://doi.org/10.1530/ EJE-16-0202

[8] MILANE L, SINGH A, MATTHEOLABAKIS G, SURESH M, AMIJI MM. Exosome mediated communication within the tumor microenvironment. J Control Release 2015; 219: 278-294. https://doi.org/10.1016/j.jconrel.2015.06.029
[9] SEO N, AKIYOSHI K, SHIKU H. Exosome-mediated regulation of tumor immunology. Cancer Sci 2018; 109: 29983004. https://doi.org/10.1111/cas. 13735

[10] JOHNSTONE RM. Exosomes biological significance: A concise review. Blood Cells Mol Dis 2006; 36: 315-321. https:// doi.org/10.1016/j.bcmd.2005.12.001

[11] CUI S, CHENG Z, QIN W, JIANG L. Exosomes as a liquid biopsy for lung cancer. Lung Cancer 2018; 116: 46-54. https://doi.org/10.1016/j.lungcan.2017.12.012

[12] SUN D, ZHUANG X, ZHANG S, DENG ZB, GRIZZLE $\mathrm{W}$ et al. Exosomes are endogenous nanoparticles that can deliver biological information between cells. Adv Drug Deliv Rev 2013; 65: 342-347. https://doi.org/10.1016/j. addr.2012.07.002

[13] LEE JC, ZHAO JT, GUNDARA J, SERPELL J, BACH LA et al. Papillary thyroid cancer-derived exosomes contain miRNA-146b and miRNA-222. J Surg Res 2015; 196: 39-48. https://doi.org/10.1016/j.jss.2015.02.027

[14] SAMSONOV R, BURDAKOV V, SHTAM T, RADZHABOVA Z, VASILYEV D et al. Plasma exosomal miR-21 and miR-181a differentiates follicular from papillary thyroid cancer. Tumour Biol 2016; 37: 12011-12021. https:// doi.org/10.1007/s13277-016-5065-3

[15] YANG H, FU H, WANG B, ZHANG X, MAO J et al. Exosomal miR-423-5p targets SUFU to promote cancer growth and metastasis and serves as a novel marker for gastric cancer. Mol Carcinog 2018; 57: 1223-1236. https://doi.org/10.1002/ mc. 22838

[16] LI S, ZENG A, HU Q, YAN W, LIU Y et al. miR-423-5p contributes to a malignant phenotype and temozolomide chemoresistance in glioblastomas. Neuro Oncol 2017; 19: 55-65. https://doi.org/10.1093/neuonc/now129

[17] MARQUES-GARCIA F, ISIDORO-GARCIA M. Protocols for Exosome Isolation and RNA Profiling. Methods Mol Biol 2016; 1434: 153-167. https://doi.org/10.1007/978-1-49393652-6_11

[18] THERY C, AMIGORENA S, RAPOSO G, CLAYTON A. Isolation and characterization of exosomes from cell culture supernatants and biological fluids. Curr Protoc Cell Biol 2006; Chapter 3: Unit 3.22. https://doi.org/10.1002/0471143030. cb0322s30

[19] KAMERKAR S, LEBLEU VS, SUGIMOTO H, YANG S, RUIVO CF et al. Exosomes facilitate therapeutic targeting of oncogenic KRAS in pancreatic cancer. Nature 2017; 546: 498-503. https://doi.org/10.1038/nature22341

[20] EL-ANDALOUSSI S, LEE Y, LAKHAL-LITTLETON S, LI J, SEOW Y et al. Exosome-mediated delivery of siRNA in vitro and in vivo. Nat Protoc 2012; 7: 2112-2126. https://doi. org/10.1038/nprot.2012.131

[21] LIU J, WANG X, YANG X, LIU Y, SHI Y et al. miRNA423$5 p$ regulates cell proliferation and invasion by targeting trefoil factor 1 in gastric cancer cells. Cancer Lett 2014; 347: 98-104. https://doi.org/10.1016/j.canlet.2014.01.024

[22] TENG Y, REN Y, HU X, MU J, SAMYKUTTY A et al. MVPmediated exosomal sorting of miR-193a promotes colon cancer progression. Nat Commun 2017; 8: 14448. https://doi. org/10.1038/ncomms 14448 
[23] SUN D, ZHUANG X, ZHANG S, DENG ZB, GRIZZLE $\mathrm{W}$ et al. Exosomes are endogenous nanoparticles that can deliver biological information between cells. Adv Drug Deliv Rev 2013; 65: 342-347. https://doi.org/10.1016/j. addr.2012.07.002
[24] FANG JH, ZHANG ZJ, SHANG LR, LUO YW, LIN YF et al. Hepatoma cell-secreted exosomal microRNA-103 increases vascular permeability and promotes metastasis by targeting junction proteins. Hepatology 2018; 68: 1459-1475. https:// doi.org/10.1002/hep.29920 